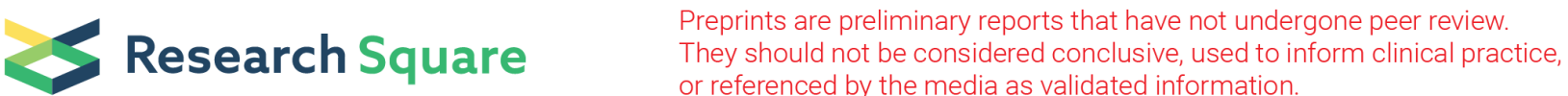

\section{Federated Learning used for predicting outcomes in SARS-COV-2 patients}

Mona Flores ( $\nabla$ mflores@nvidia.com )

NVIDIA https://orcid.org/0000-0002-7362-3044

\section{Ittai Dayan}

MGH Radiology and Harvard Medical School

\section{Holger Roth}

NVIDIA https://orcid.org/0000-0002-3662-8743

\section{Aoxiao Zhong}

Center for Advanced Medical Computing and Analysis, Department of Radiology, Massachusetts General Hospital, Harvard Medical School, Boston, MA

\section{Ahmed Harouni}

NVIDIA

\section{Amilcare Gentili}

San Diego VA Health Care System, San Diego

\section{Anas Abidin}

NVIDIA

\section{Andrew Liu}

andrliu@nvidia.com

\section{Anthony Costa}

Mount Sinai Health System

\section{Bradford Wood}

Radiology \& Imaging Sciences / Clinical Center, National Institutes of Health

\section{Chien-Sung Tsai}

Division of Cardiovascular Surgery, Department of Surgery, Tri-Service General Hospital, National

Defense Medical Center, Taipei, Taiwan, R.O.C.

\section{Chih-Hung Wang}

Tri-Service General Hospital, National Defense Medical Center https://orcid.org/0000-0001-5058-4356

\section{Chun-Nan Hsu}

Center for Research in Biological Systems, University of California, San Diego https://orcid.org/00000002-5240-4707

\section{CK Lee}

NVIDIA

\section{Colleen Ruan}

NVIDIA 


\section{Daguang Xu}

NVIDIA

\section{Dufan Wu}

Center for Advanced Medical Computing and Analysis, Department of Radiology, Massachusetts General Hospital, Harvard Medical School, Boston, MA

\section{Eddie Huang}

NVIDIA

\section{Felipe Kitamura}

Diagnósticos da América SA (Dasa) https://orcid.org/0000-0002-9992-5630

\section{Griffin Lacey}

NVIDIA

\section{Gustavo César de Antônio Corradi}

GUSTAVOCORRADI@gmail.com

\section{Hao-Hsin Shin}

Memorial Sloan Kettering Cancer Center

\section{Hirofumi Obinata}

Self-Defense Forces Central Hospital

\section{Hui Ren}

Center for Advanced Medical Computing and Analysis, Department of Radiology, Massachusetts General Hospital, Harvard Medical School, Boston, MA

\section{Jason Crane}

Center for Intelligent Imaging, Department of Radiology and Biomedical Imaging, University of California, San Francisco, California, USA.

\section{Jesse Tetreault}

NVIDIA

\section{Jiahui Guan}

NVIDIA

\section{John Garrett}

The University of Wisconsin-Madison School of Medicine and Public Health https://orcid.org/00000002-8152-736X

\section{Jung Gil Park}

Yeungnam University College of Medicine https://orcid.org/0000-0001-5472-4731

\section{Keith Dreyer}

Center for Clinical Data Science, Massachusetts General Brigham, Boston, MA

\section{Krishna Juluru}

Memorial Sloan Kettering Cancer Center https://orcid.org/0000-0001-8203-8894

\section{Kristopher Kersten}

NVIDIA

\section{Marcio Aloisio Bezerra Cavalcanti Rockenbach}


Center for Clinical Data Science, Massachusetts General Brigham, Boston, MA https://orcid.org/00000003-1783-0441

\section{Marius Linguraru}

Sheikh Zayed Institute for Pediatric Surgical Innovation, Children's National Hospital and School of Medicine and Health Sciences, George Washington University, Washington, DC

\section{Masoom Haider}

Joint Dept. of Medical Imaging, Sinai Health System, University of Toronto, Toronto, Canada and Lunenfeld-Tanenbaum Research Institute, Toronto, Canada

\section{Meena AbdelMaseeh}

Lunenfeld-Tanenbaum Research Institute, Toronto, Canada

\section{Nicola Rieke}

NVIDIA

\section{Pablo Damasceno}

Center for Intelligent Imaging, Department of Radiology and Biomedical Imaging, University of California, San Francisco, California, USA.

\section{Pedro Mario Cruz e Silva}

NVIDIA

\section{Pochuan Wang}

MeDA Lab and Institute of Applied Mathematical Sciences, National Taiwan University, Taipei, Taiwan https://orcid.org/0000-0002-3856-048X

\section{Sheng Xu}

Center for Interventional Oncology, National Institutes of Health, Bethesda, MD, USA

\section{Shuichi Kawano}

Self-Defense Forces Central Hospital

\section{Sira Sriswasdi}

Chulalongkorn University https://orcid.org/0000-0002-4117-3632

\section{Soo Young Park}

Department of Internal Medicine, School of Medicine, Kyungpook National University, Daegu, South Korea

\section{Thomas Grist}

University of Wisconsin-Madison

\section{Varun Buch}

Center for Clinical Data Science, Massachusetts General Brigham, Boston, MA

\section{Watsamon Jantarabenjakul}

Department of Pediatrics, Faculty of Medicine, Chulalongkorn University, Bangkok, Thailand and Thai Red Cross Emerging Infectious Diseases Clinical Center, King Chulalongkorn Memorial Hospital, Bang

\section{Weichung Wang}

National Taiwan University

\section{Won Young Tak}


Department of Internal Medicine, School of Medicine, Kyungpook National University, Daegu, South Korea

\section{Xiang Li}

Center for Advanced Medical Computing and Analysis, Department of Radiology, Massachusetts

General Hospital, Harvard Medical School, Boston, MA

\section{Xihong Lin}

Harvard T.H. Chan School of Public Health https://orcid.org/0000-0001-7067-7752

\section{Fred Kwon}

Mount Sinai Health System

\section{Fiona Gilbert}

University of Cambridge https://orcid.org/0000-0002-0124-9962

\section{Josh Kaggie}

Department of Radiology, NIHR Cambridge Biomedical Resource Centre, University of Cambridge

\section{Quanzheng Li}

Center for Advanced Medical Computing and Analysis, Department of Radiology, Massachusetts General Hospital, Harvard Medical School, Boston, MA

\section{Abood Quraini}

NVIDIA

\section{Andrew Feng}

NVIDIA

\section{Andrew Priest}

Department of Radiology, NIHR Cambridge Biomedical Resource Centre, Cambridge University Hospital https://orcid.org/0000-0002-9771-4290

\section{Baris Turkbey}

National Institutes of Health https://orcid.org/0000-0003-0853-6494

\section{Benjamin Glicksberg}

Icahn School of Medicine at Mount Sinai https://orcid.org/0000-0003-4515-8090

\section{Bernardo Bizzo}

Center for Clinical Data Science, Massachusetts General Brigham, Boston, MA https://orcid.org/00000002-9686-6751

\section{Byung Seok Kim}

Department of Internal Medicine, Catholic University of Daegu School of Medicine, Daegu, South Korea

\section{Carlos Tor-Diez}

Sheikh Zayed Institute for Pediatric Surgical Innovation, Children's National Hospital , Washington, DC https://orcid.org/0000-0003-3339-5777

\section{Chia-Cheng Lee}

Planning and Management Office, Tri-Service General Hospital, National Defense Medical Center, Taipei, Taiwan, R.O.C. and Division of Colorectal Surgery, Department of Surgery, Tri-Service General H

\section{Chia-Jung Hsu}


Planning and Management Office, Tri-Service General Hospital, National Defense Medical Center, Taipei, Taiwan, R.O.C.

\section{Chin Lin}

School of Medicine, National Defense Medical Center, Taipei, Taiwan, R.O.C. and School of Public Health, National Defense Medical Center, Taipei, Taiwan, R.O.C. and Graduate Institute of Life Scienc

\section{Chiu-Ling Lai}

Medical Review and Pharmaceutical Benefits Division, National Health Insurance Administration, Taipei.

\section{Taiwan}

\section{Christopher Hess}

University of California, San Francisco

\section{Colin Compas}

NVIDIA

\section{Deepi Bhatia}

NVIDIA

\section{Eric Oermann}

NYU Langone

\section{Evan Leibovitz}

The Center for Clinical Data Science, Mass General Brigham.

\section{Hisashi Sasaki}

Self-Defense Forces Central Hospital

\section{Hitoshi Mori}

Self-Defense Forces Central Hospital

\section{Isaac Yang}

NVIDIA

\section{Jae Ho Sohn}

Center for Intelligent Imaging, Department of Radiology and Biomedical Imaging, University of California, San Francisco, California, USA.

\section{Krishna Nand Keshava Murthy}

Memorial Sloan Kettering Cancer Center

\section{Li-Chen Fu}

MOST/NTU All Vista Healthcare Center, Center for Artificial Intelligence and Advanced Robotics, National Taiwan University, Taipei, Taiwan

\section{Matheus Ribeiro Furtado de Mendonça}

Diagnósticos da América SA (DASA) https://orcid.org/0000-0001-5541-7207

\section{Mike Fralick}

Division of General Internal Medicine and Geriatrics (Fralick), Sinai Health System, Toronto, Canada

\section{Min Kyu Kang}

Department of Internal Medicine, Yeungnam University College of Medicine, Daegu, South Korea

\section{Mohammad Adil}


NVIDIA

\section{Natalie Gangai}

Memorial Sloan Kettering Cancer Center

\section{Peerapon Vateekul}

Department of Computer Engineering, Faculty of Engineering, Chulalongkorn University https://orcid.org/0000-0001-9718-3592

\section{Pierre Elnajjar}

Memorial Sloan Kettering Cancer Center

\section{Sarah Hickman}

Department of Radiology, NIHR Cambridge Biomedical Resource Centre, University of Cambridge

\section{Sharmila Majumdar}

Center for Intelligent Imaging, Department of Radiology and Biomedical Imaging, University of California, San Francisco, California, USA.

\section{Shelley McLeod}

Schwartz/Reisman Emergency Medicine Institute, Sinai Health, Toronto, ON, Canada and Department of Family and Community Medicine, University of Toronto, Toronto, ON, Canada

\section{Sheridan Reed}

Center for Interventional Oncology, National Institutes of Health, Bethesda, MD, USA

\section{Stefan Graf}

University of Cambridge https://orcid.org/0000-0002-1315-8873

\section{Stephanie Harmon}

National Cancer Institute https://orcid.org/0000-0002-2507-2399

\section{Tatsuya Kodama}

Self-Defense Forces Central Hospital

\section{Thanyawee Puthanakit}

Department of Pediatrics, Faculty of Medicine, Chulalongkorn University, Center of Excellence in Pediatric Infectious Diseases and Vaccine, Chulalongkorn University

\section{Tony Mazzulli}

Department of Microbiology, Sinai Health/University Health Network, Toronto, Canada and Department of Laboratory Medicine and Pathobiology, University of Toronto, Toronto. Canada Public Health Ontar

\section{Vitor de Lima Lavor}

Diagnósticos da América SA (DASA)

\section{Yothin Rakvongthai}

Chulalongkorn University Biomedical Imaging Group and Division of Nuclear Medicine, Department of Radiology, Faculty of Medicine, Chulalongkorn University, Bangkok, Thailand

\section{Yu Rim Lee}

Department of Internal Medicine, School of Medicine, Kyungpook National University, Daegu, South Korea

\section{Yuhong Wen}




\section{Article}

Keywords: federated learning, artificial intelligence, SARS-COV-2

Posted Date: January 8th, 2021

DOI: https://doi.org/10.21203/rs.3.rs-126892/v1

License: (c) (1) This work is licensed under a Creative Commons Attribution 4.0 International License. Read Full License

Version of Record: A version of this preprint was published at Nature Medicine on September 15th, 2021. See the published version at https://doi.org/10.1038/s41591-021-01506-3. 


\section{Abstract}

'Federated Learning' (FL) is a method to train Artificial Intelligence (AI) models with data from multiple sources while maintaining anonymity of the data thus removing many barriers to data sharing. During the SARS-COV-2 pandemic, 20 institutes collaborated on a healthcare FL study to predict future oxygen requirements of infected patients using inputs of vital signs, laboratory data, and chest x-rays, constituting the "EXAM" (EMR CXR Al Model) model. EXAM achieved an average Area Under the Curve (AUC) of over 0.92 , an average improvement of $16 \%$, and a $38 \%$ increase in generalisability over local models. The FL paradigm was successfully applied to facilitate a rapid data science collaboration without data exchange, resulting in a model that generalised across heterogeneous, unharmonized datasets. This provided the broader healthcare community with a validated model to respond to COVID19 challenges, as well as set the stage for broader use of FL in healthcare.

\section{Main Text}

The scientific and academic medical and data science communities have come together in the face of the pandemic crisis in order to rapidly assess novel paradigms in artificial intelligence that are rapid and secure, and potentially incentivize data sharing and model training and testing without the usual privacy and data ownership hurdles of conventional collaborations ${ }^{1,2}$. Healthcare providers, researchers and industry have pivoted their focus to address unmet and critical clinical needs created by the crisis, with remarkable results ${ }^{3-6}$. Clinical trial recruitment has been expedited and facilitated by national regulatory bodies and an international cooperative spirit ${ }^{7-9}$. The data analytics and artificial intelligence (Al) disciplines have always fostered open and collaborative approaches, embracing concepts such as opensource software, reproducible research, data repositories, and making anonymized datasets publicly available ${ }^{10,11}$. The pandemic has emphasized the need to expeditiously conduct data collaborations that empower the clinical and scientific communities when responding to rapidly evolving and widespread global challenges. Data sharing has ethical, regulatory and legal complexities that are underscored, and perhaps somewhat complicated by the recent entrance of large tech companies into the healthcare data world ${ }^{12-15}$.

A concrete example for these types of collaborations is our recent work on an Al-based SARS-COV-2 Clinical Decision Support (CDS) algorithm. The CDS predicts a risk score that can be used to support decisions to admit infected patients to the hospital and to help determine the level of hospital care they will likely require. We refined and validated the algorithm across multiple health systems. The CDS was created at Mass General Brigham (MGB), using chest x-ray (CXR) data, vital signs, demographic data, and lab values that were shown to be predictive of COVID-19 patient outcomes ${ }^{16-1816-19}$. The CDS outputs a score, 'CORISK', that predicts oxygen support requirement, and can be used as a decision aid tool for triaging patients by front-line clinicians ${ }^{20-22}$. 
Healthcare providers have preferred using algorithms that were validated on their own data ${ }^{23}$. To date, most Al algorithms have been trained and validated only on a few datasets that often lacked in diversity 24,25 , resulting in less generalisable performance. Even near-perfect peer-reviewed performance metrics do not guarantee generalisability nor a lack of over-fitting. Our aim was to develop an algorithm trained on a diverse dataset, making it useful, trusted and generalisable across a large number of healthcare systems. Accessing diverse data without the requirement of centralised data ${ }^{26}$ is enabled by techniques such as Transfer Learning ${ }^{27}$ and 'Federated Learning' (FL) ${ }^{28}$ for achieving distributed model training and validation. The authors chose FL due to its ability to rapidly launch centrally orchestrated experiments with improved traceability of data and assessment of algorithmic changes and impact ${ }^{29}$. FL has shown promise in recent medical imaging applications ${ }^{30-33}$, including COVID-19 analysis ${ }^{34-37}$, albeit with limited scale. Governance of data for FL is maintained locally, alleviating privacy concerns, with only model 'weights' or 'gradients' transferred between the client-sites and the federated server ${ }^{38,39}$.

Driven by the pandemic and enabled by the privacy-conserving nature of FL, 20 institutions were recruited, the majority of which were hospitals. The study named "EXAM" (EMR Chest X-Ray Al Model), consisted of algorithm development by a Mass General Brigham team during March 2020, and the recruitment for this FL study that started in June. Between August and October, 140 experiments were conducted, and by end-October 2020, the refined version of the algorithm was made public on NVIDIA $\mathrm{NGC}^{40}$.

\section{A global dataset for COVID-19 image analysis}

The 20 client-sites prepared 16,148 cases (both positive and negative) for the purpose of training, validating, and testing the model. Each case included one CXR and the requisite data inputs taken from the patient's medical record. A breakdown of the cohort size of the dataset for each client site is shown in Fig. 1b. The significant diversity of data between sites motivated the researchers in creating the dataset, since capturing these differences was thought to be needed in order to create a performant CDS. The distribution and patterns of CXR image intensities (pixel values) varied significantly among the sites due to a multitude of patient and site-specific factors, such as differences in device manufacturers and imaging protocols, as shown in Fig. 1c. Patient age and EMR data varied for different sites due to the demographic differences between hospitals located around the globe (Fig. 1d and extended Data Fig. 1).

\section{An Al model to predict a 'CORISK' score}

There is wide variation in the clinical course of patients who present to the hospital with symptoms of COVID-19, with some experiencing rapid deterioration in respiratory function requiring different interventions in order to prevent or mitigate hypoxemia ${ }^{41,42}$. A critical decision made during the evaluation of a patient at the initial point of care or the ED, is whether the patient is likely to require more invasive or resource-limited counter-measures or interventions (such as mechanical ventilation or monoclonal antibodies), and should therefore receive a scarce but effective therapy, a therapy with a narrow risk-benefit ratio due to side effects, or a higher level of care, such as admittance to the ICU ${ }^{43,44}$. In 
contrast, a patient who is at a lower risk of requiring invasive oxygen therapy may be placed in a less intensive care setting such as a regular ward or even released from the ED for continued self-monitoring at home ${ }^{45}$.

Therefore, the model was trained to predict the 'CORISK' score corresponding to a patient's oxygen needs within two prediction windows, 24 hours and 72 hours after initial presentation to the ED. We set the outcome labels of patients as $0,0.25,0.5$, and 0.75 if the most intensive oxygen therapy the patient received in the prediction window was room air (RA), low-flow oxygen (LFO), high-flow oxygen (HFO)/noninvasive ventilation (NIV), or mechanical ventilation (MV), respectively. If the patient died within the prediction window, the outcome label was set to 1 . This resulted in each case being assigned two labels in the range of 0 to 1, corresponding to each of the prediction windows. For EMR features, data preprocessing included de-identification, missing value imputation (using the MissForest algorithm ${ }^{46}$ ), and normalization to zero-mean and unit variance. CXR images were preprocessed to select the right series and exclude lateral view images, then scaled to a resolution of $224 \times 224$. As shown in Fig. 2 , the model fuses information from both the EMR features and CXR features (based on a modified ResNet-34

with spatial attention ${ }^{47,48}$ pre-trained on the CheXpert dataset ${ }^{49}$, and Deep \& Cross network ${ }^{50}$. In order to converge these different data types, a 512-dimensional feature vector was extracted from each CXR image using a pre-trained ResNet-34, with spatial attention, then concatenated with the EMR features as the input for the Deep \& Cross network (see Methods). The final output was a continuous value from 0 to 1 for both the 24 hour and 72-hour predictions, corresponding to the labels described above. We used binary cross-entropy as the loss function and 'Adam' as the optimizer. The model was implemented in Tensorflow ${ }^{51}$ using the NVIDIA Clara Train SDK ${ }^{52}$. The average AUC for the three prediction tasks (LFO, HFO/NIV, or MV) was calculated and used as the final evaluation metric (see Methods).

\section{Performance boosts through Federated Learning}

Arguably, the most established form of FL is implementing the Federated Averaging algorithm proposed by McMahan et al $^{53}$, or variations thereof. This algorithm can be realised using a client-server setup, where each participating site acts as a client. One can think of FL as a method aiming to minimize a global loss function by reducing a set of local loss functions, which are estimated at each site. By minimizing each client site's local loss while also synchronizing the learned client site weights on a centralized aggregation server, one can minimize the global loss without needing to access the entire dataset in a centralized location. Each client site learns locally, and shares model weight updates with a central server that aggregates contributions using secure SSL encryption and communication protocols ${ }^{54}$. The server then sends an updated set of weights to each client site after the aggregation, and sites resume training locally. The server and client site iterate back and forth until the model converges (see Methods section). To analyse the stability of these results, we repeated three runs of local training and FL on different randomized data splits. Training the model through FL resulted in a significant performance improvement ( $p<<1 e-3$, Wilcoxon signed-rank test) of $16 \%$ (as defined by the average-AUC when running the model on respective local test sets) and a $38 \%$ generalisability improvement (as defined by the average-AUC when running the model on all test sets) of the final global model for predicting $24 \mathrm{~h}$ 
oxygen treatment compared to models trained only on a site's own data (Fig. 3). The results for predicting $72 \mathrm{~h}$ oxygen treatments are shown in Extended Data Fig. 7 and resulted in a performance improvement of $18 \%$ compared to locally trained models alone, while generalisability of the global model improved by $34 \%$.

\section{Security Considerations}

A primary motivation for healthcare institutes to use FL is to preserve the security and privacy of their data, as well as adhere to data compliance measures. However, there remains a potential risk of model 'inversion' ${ }^{55}$ or even reconstructing training images from the model gradients themselves ${ }^{56}$. To counter these risks, there are security-enhancing measures that may be able to mitigate risk in the event of data 'interception' during site-server communication ${ }^{57}$. We investigated a partial weight-sharing scheme ${ }^{58,59}$ showing that models can reach a comparable performance even when only $25 \%$ of the weight updates are shared (Fig. 4 and Methods section). The weight updates were ranked during each iteration by magnitude of contribution and only a certain percentage of the largest weight updates were shared with the server (see Methods). With this, we validated previous findings, showing that partial weight sharing, and other differential privacy techniques can successfully be applied in $\mathrm{FL}^{58}$.

\section{Impact on patient care}

To our knowledge, this study features the largest real-world healthcare FL experiment to date in terms of number of sites and number of data points used. The study encompassed 20 client-sites and included over 16,000 cases (Extended Data Table 2). We believe that it provides a powerful case study for the utilization of FL involving multiple sites across 5 continents and under the supervision of different regulatory bodies. The global algorithm proved to be more robust and achieved better results on individual sites than any model that was trained on local data. We believe that the consistent improvement was achieved not only due to a larger, but also a more diverse data set.

We observed that FL improved the prediction accuracy on all site testing sets, even when sites had relatively large local training data sets. For sites with small datasets, it was virtually impossible to build a performant deep learning model using only their local data. Furthermore, sites whose local models were trained with unbalanced cohorts (e.g., with most subjects experiencing mild cases of COVID-19) markedly benefited from the FL approach (Extended Data Figs. 3 \& 4). More importantly, the generalisability of the FL model increased considerably, over the locally trained model, most likely since a population or an age group that are under-represented in one hospital/region could be highly represented in another region (Extended Data Figs. 5 \& 6 and Extended Data Table 3). For example, children might be differentially affected by COVID-19, including their manifestations in lung imaging ${ }^{60}$.

As seen in Fig. 1c/d and Extended Data Fig. 1, we designed our study to resemble real-life clinical situations by intentionally not completing a meticulous harmonization of the data inputs. The features derived from the medical record were carefully defined in order to mitigate potential biases (Extended Data Table 1). Features that were expected to be influenced by different clinical practices and standards 
of care were avoided, such as reported symptoms or clinical impressions. We also chose model outputs that we believed to be objective outcomes which are fairly practical to discern, being low-flow oxygen treatment, high-flow oxygen treatment, mechanical ventilation, and death (Extended Data Fig. 2). We believe that these design considerations played a significant part in increasing the benefits from a FL approach and its impact on model performance, generalisability, and ultimately, its usability. By participating in this study, the client-sites received access to an optimized Al model ('global FL model'), that can be further validated ahead of introduction into clinical care. The client-sites did not transfer data to a central repository but rather created a distributed data framework that can facilitate ongoing collaboration on Al model development and validation. We believe that the preservation of privacy, afforded by FL, encouraged participation of institutes who recognized the urgency to contribute during the COVID pandemic, and were not held back by data governance constraints. As mentioned above, we also experimented with techniques to avoid 'interception' of FL data, and found them to be promising (Fig. 4). This is an added security feature that we believe will encourage more institutions to use FL.

\section{Future development and outlook}

In the opinion of this group, the main areas for development arising out of this collaboration will be to streamline data access, preparation and methods in order to better leverage a network of sites participating in FL. A system that would allow real-time model inference and processing would also be of benefit and would 'close the loop' from training to model deployment. Patient cohort identification and data harmonization are not new issues in research and data science ${ }^{61}$, but are further complicated given the lack of visibility on other sites' data sets associated with FL. There is also a need for evolving our understanding of architectural considerations that will enable capturing more value out of FL, e.g., explicitly addressing the data domain shifts between the different participating sites ${ }^{62}$. Hyperparameter engineering can allow algorithms to 'learn' more effectively from larger data batches and adapt model parameters to a particular site for further personalization. For example, socio-economic status or ethnicity in an algorithm prototyped on a homogenous population could enable algorithms to capture more diversity in FL training, despite being less meaningful when only leveraging a single-site data set. Additionally, there is a need to improve our ability to predict each client-site's contribution to improving the global FL model, which will help in client-site selection and prioritizing data acquisition and annotation efforts in the future. The latter is especially important given the high costs and difficult logistics of these large consortia endeavors, and the opportunity to capture diversity rather than sheer quantity of data samples.

\section{References}

1. Budd, J. et al. Digital technologies in the public-health response to COVID-19. Nat. Med. 26, 1183$1192(2020)$.

2. Moorthy, V., Henao Restrepo, A. M., Preziosi, M.-P. \& Swaminathan, S. Data sharing for novel coronavirus (COVID-19). Bull. World Health Organ. 98, 150 (2020).

3. Chen, Q., Allot, A. \& Lu, Z. Keep up with the latest coronavirus research. Nature 579, 193-193 (2020). 
4. The Impact of the COVID-19 Pandemic on Outpatient Care: Visits Return to Prepandemic Levels, but Not for All Providers and Patients.

https://www.commonwealthfund.org/publications/2020/oct/impact-covid-19-pandemic-outpatientcare-visits-return-prepandemic-levels doi:10.26099/41xy-9m57.

5. Fabbri, F., Bhatia, A., Mayer, A., Schlotter, B. \& Kaiser, J. BCG IT Spend Pulse: How COVID-19 Is Shifting Tech Priorities. https://www.bcg.com/publications/2020/how-covid-19-is-shifting-big-itspend (2020).

6. Candelon, F., Reichert, T., Duranton, S., di Carlo, R. C. \& De Bondt, M. The Rise of the Al-Powered Company in the Postcrisis World. https://www.bcg.com/publications/2020/business-applicationsartificial-intelligence-post-covid (2020).

7. COVID-19 Studies from the World Health Organization Database. https://clinicaltrials.gov/ct2/who_table.

8. ACTIV. https://www.nih.gov/research-training/medical-research-initiatives/activ.

9. Center for Drug Evaluation \& Research. Coronavirus Treatment Acceleration Program (CTAP). https://www.fda.gov/drugs/coronavirus-covid-19-drugs/coronavirus-treatment-acceleration-programctap (2020).

10. Gleeson, P., Davison, A. P., Silver, R. A. \& Ascoli, G. A. A Commitment to Open Source in Neuroscience. Neuron 96, 964-965 (2017).

11. Piwowar, H. et al. The state of OA: a large-scale analysis of the prevalence and impact of Open Access articles. PeerJ 6, e4375 (2018).

12. European Society of Radiology (ESR). What the radiologist should know about artificial intelligence an ESR white paper. Insights into Imaging vol. 10 (2019).

13. Pesapane, F., Volonté, C., Codari, M. \& Sardanelli, F. Artificial intelligence as a medical device in radiology: ethical and regulatory issues in Europe and the United States. Insights Imaging 9, 745753 (2018).

14. Price, W. N., 2nd \& Cohen, I. G. Privacy in the age of medical big data. Nat. Med. 25, 37-43 (2019).

15. Cohen, I. G., Amarasingham, R., Shah, A., Xie, B. \& Lo, B. The legal and ethical concerns that arise from using complex predictive analytics in health care. Health Aff. 33, 1139-1147 (2014).

16. Liang, W. et al. Development and Validation of a Clinical Risk Score to Predict the Occurrence of Critical IIIness in Hospitalized Patients With COVID-19. JAMA Intern. Med. 180, 1081-1089 (2020).

17. Wynants, L. et al. Prediction models for diagnosis and prognosis of covid-19 infection: systematic review and critical appraisal. BMJ 369, m1328 (2020).

18. Zhang, L. et al. D-dimer levels on admission to predict in-hospital mortality in patients with Covid-19. J. Thromb. Haemost. 18, 1324-1329 (2020).

19. Sands, K. E. et al. Patient characteristics and admitting vital signs associated with coronavirus disease 2019 (COVID-19)-related mortality among patients admitted with noncritical illness. Infect. Control Hosp. Epidemiol. 1-7 (2020). 
20. Website. https://doi.org/10.1111/anae.15073 doi:10.1111/anae.15073.

21. Whittle, J. S., Pavlov, I., Sacchetti, A. D., Atwood, C. \& Rosenberg, M. S. Respiratory support for adult patients with COVID-19. J Am Coll Emerg Physicians Open (2020) doi:10.1002/emp2.12071.

22. Ai, J., Li, Y., Zhou, X. \& Zhang, W. COVID-19: treating and managing severe cases. Cell Res. 30, 370371 (2020).

23. Esteva, A. et al. A guide to deep learning in healthcare. Nat. Med. 25, 24-29 (2019).

24. Cahan, E. M., Hernandez-Boussard, T., Thadaney-Israni, S. \& Rubin, D. L. Putting the data before the algorithm in big data addressing personalized healthcare. npj Digital Medicine 2, 1-6 (2019).

25. Thrall, J. H. et al. Artificial Intelligence and Machine Learning in Radiology: Opportunities, Challenges, Pitfalls, and Criteria for Success. J. Am. Coll. Radiol. 15, 504-508 (2018).

26. Shilo, S., Rossman, H. \& Segal, E. Axes of a revolution: challenges and promises of big data in healthcare. Nat. Med. 26, 29-38 (2020).

27. Gao, Y. \& Cui, Y. Deep transfer learning for reducing health care disparities arising from biomedical data inequality. Nat. Commun. 11, 5131 (2020).

28. Yang, Q., Liu, Y., Chen, T. \& Tong, Y. Federated Machine Learning: Concept and Applications. (2019).

29. Rieke, N. et al. The future of digital health with federated learning. NPJ Digit Med 3, 119 (2020).

30. Roth, H. R. et al. Federated Learning for Breast Density Classification: A Real-World Implementation: Second MICCAI Workshop, DART 2020, and First MICCAI Workshop, DCL 2020, Held in Conjunction with MICCAI 2020, Lima, Peru, October 4-8, 2020, Proceedings. in Domain Adaptation and Representation Transfer, and Distributed and Collaborative Learning (eds. Albarqouni, S. et al.) vol. 12444 181-191 (Springer International Publishing, 2020).

31. Sheller, M. J. et al. Federated learning in medicine: facilitating multi-institutional collaborations without sharing patient data. Sci. Rep. 10, 12598 (2020).

32. Remedios, S. W., Butman, J. A., Landman, B. A. \& Pham, D. L. Federated Gradient Averaging for MultiSite Training with Momentum-Based Optimizers. in Domain Adaptation and Representation Transfer, and Distributed and Collaborative Learning 170-180 (Springer International Publishing, 2020).

33. Wang, P. et al. Automated Pancreas Segmentation Using Multi-institutional Collaborative Deep Learning. in Domain Adaptation and Representation Transfer, and Distributed and Collaborative Learning 192-200 (Springer International Publishing, 2020).

34. Xu, Y. et al. A collaborative online Al engine for CT-based COVID-19 diagnosis. medRxiv (2020) doi:10.1101/2020.05.10.20096073.

35. Raisaro, J. L. et al. SCOR: A secure international informatics infrastructure to investigate COVID-19. Journal of the American Medical Informatics Association (2020) doi:10.1093/jamia/ocaa172.

36. Vaid, A. et al. Federated Learning of Electronic Health Records Improves Mortality Prediction in Patients Hospitalized with COVID-19. medRxiv (2020) doi:10.1101/2020.08.11.20172809.

37. Yang, D. et al. Federated Semi-Supervised Learning for COVID Region Segmentation in Chest CT using Multi-National Data from China, Italy, Japan. arXiv [eess.IV] (2020). 
38. Ma, C. et al. On Safeguarding Privacy and Security in the Framework of Federated Learning. IEEE Netw. 34, 242-248 (2020).

39. Brisimi, T. S. et al. Federated learning of predictive models from federated Electronic Health Records. Int. J. Med. Inform. 112, 59-67 (2018).

40. COVID-19 Related Models. NVIDIA GPU Cloud (NGC) Platform https://ngc.nvidia.com/catalog/models? orderBy=scoreDESC\&pageNumber=0\&query=covid\&quickFilter=models\&filters=.

41. Navas-Blanco, J. R. \& Dudaryk, R. Management of Respiratory Distress Syndrome due to COVID-19 infection. BMC Anesthesiol. 20, 177 (2020).

42. Marini, J. J. \& Gattinoni, L. Management of COVID-19 Respiratory Distress. JAMA 323, 2329-2330 (2020).

43. Cook, T. M. et al. Consensus guidelines for managing the airway in patients with COVID-19: Guidelines from the Difficult Airway Society, the Association of Anaesthetists the Intensive Care Society, the Faculty of Intensive Care Medicine and the Royal College of Anaesthetists. Anaesthesia 75, 785-799 (2020).

44. Galloway, J. B. et al. A clinical risk score to identify patients with COVID-19 at high risk of critical care admission or death: An observational cohort study. J. Infect. 81, 282-288 (2020).

45. Kilaru, A. S. et al. Return Hospital Admissions Among 1419 COVID-19 Patients Discharged from Five U.S. Emergency Departments. Acad. Emerg. Med. 27, 1039-1042 (2020).

46. Stekhoven, D. J. \& BühImann, P. MissForest-non-parametric missing value imputation for mixed-type data. Bioinformatics 28, 112-118 (2012).

47. He, K., Zhang, X., Ren, S. \& Sun, J. Deep Residual Learning for Image Recognition. 2016 IEEE Conference on Computer Vision and Pattern Recognition (CVPR) (2016) doi:10.1109/cvpr.2016.90.

48. Zhong, A. et al. Deep Metric Learning-based Image Retrieval System for Chest Radiograph and its Clinical Applications in COVID-19. arXiv [eess.IV] (2020).

49. Irvin, J. et al. CheXpert: A Large Chest Radiograph Dataset with Uncertainty Labels and Expert Comparison. Proceedings of the AAAI Conference on Artificial Intelligence vol. 33 590-597 (2019).

50. Wang, R., Fu, B., Fu, G. \& Wang, M. Deep \& Cross Network for Ad Click Predictions. Proceedings of the ADKDD'17 on ZZZ - ADKDD'17(2017) doi:10.1145/3124749.3124754.

51. Martın Abadi, Paul Barham, Jianmin Chen, Zhifeng Chen, Andy Davis, Jeffrey Dean, Matthieu Devin, Sanjay Ghemawat, Geoffrey Irving, Michael Isard, Manjunath Kudlur, Josh Levenberg, Rajat Monga, Sherry Moore, Derek G. Murray, Benoit Steiner, Paul Tucker, Vijay Vasudevan, Pete Warden, Martin Wicke, Yuan Yu, and Xiaoqiang Zheng. Tensorflow: A system for large-scale machine learning. in 12th USENIX Symposium on Operating Systems Design and Implementation (2016).

doi:10.1007/978-1-4842-6699-1_1.

52. NVIDIA Clara Imaging. https://developer.nvidia.com/clara-medical-imaging. 
53. McMahan, B., Moore, E., Ramage, D., Hampson, S. \& y Arcas, B. A. Communication-Efficient Learning of Deep Networks from Decentralized Data. in (eds. Singh, A. \& Zhu, J.) vol. 54 1273-1282 (PMLR, 2017).

54. Wen, Y. et al. Federated Learning powered by NVIDIA Clara. https://developer.nvidia.com/blog/federated-learning-clara/ (2019).

55. Fredrikson, M., Jha, S. \& Ristenpart, T. Model Inversion Attacks that Exploit Confidence Information and Basic Countermeasures. in Proceedings of the 22nd ACM SIGSAC Conference on Computer and Communications Security 1322-1333 (Association for Computing Machinery, 2015).

56. Zhu, L., Liu, Z. \& Han, S. Deep Leakage from Gradients. in Advances in Neural Information Processing Systems 32 (eds. Wallach, H. et al.) 14774-14784 (Curran Associates, Inc., 2019).

57. Kaissis, G. A., Makowski, M. R., Rückert, D. \& Braren, R. F. Secure, privacy-preserving and federated machine learning in medical imaging. Nature Machine Intelligence vol. 2 305-311 (2020).

58. Li, W. et al. Privacy-Preserving Federated Brain Tumour Segmentation. Machine Learning in Medical Imaging 133-141 (2019) doi:10.1007/978-3-030-32692-0_16.

59. Shokri, R. \& Shmatikov, V. Privacy-preserving deep learning. 2015 53rd Annual Allerton Conference on Communication, Control, and Computing (Allerton) (2015) doi:10.1109/allerton.2015.7447103.

60. Nino, G. et al. Pediatric lung imaging features of COVID-19: A systematic review and meta-analysis. Pediatr. Pulmonol. 8, e201346 (2020).

61. Jiang, G. et al. Harmonization of detailed clinical models with clinical study data standards. Methods Inf. Med. 54, 65-74 (2015).

62. Li, X. et al. Multi-site fMRI analysis using privacy-preserving federated learning and domain adaptation: ABIDE results. Med. Image Anal. 65, 101765 (2020).

63. Li, X., Huang, K., Yang, W., Wang, S. \& Zhang, Z. On the Convergence of FedAvg on Non-IID Data. arXiv [stat.ML](2019).

64. Ioffe, S. \& Szegedy, C. Batch Normalization: Accelerating Deep Network Training by Reducing Internal Covariate Shift. arXiv [cs.LG](2015).

65. Hsieh, K., Phanishayee, A., Mutlu, O. \& Gibbons, P. B. The Non-IID Data Quagmire of Decentralized Machine Learning. arXiv [cs.LG](2019).

66. Robin, X. et al. pROC: an open-source package for R and S+ to analyze and compare ROC curves. BMC Bioinformatics 12, 77 (2011).

67. Yang, D. et al. Searching Learning Strategy with Reinforcement Learning for 3D Medical Image Segmentation. in Medical Image Computing and Computer Assisted Intervention - MICCAI 2019311 (Springer International Publishing, 2019).

68. Elsken, T., Metzen, J. H. \& Hutter, F. Neural Architecture Search: A Survey. arXiv [stat.ML](2018).

69. Yao, Q. et al. Taking Human out of Learning Applications: A Survey on Automated Machine Learning. $\operatorname{arXiv}[$ cs.Al] (2018).

70. Xie, C., Koyejo, S. \& Gupta, I. Asynchronous Federated Optimization. arXiv [cs.DC] (2019). 


\section{Methods}

\section{Ethics approval}

All procedures were conducted in accordance with principles for human experimentation as defined in the Declaration of Helsinki and International Conference on Harmonization Good Clinical Practice guidelines and approved by the relevant institutional review boards (e.g., the Mass General Brigham ethics board, reference \# 2020P002673). Since no patient data was transferred between any of the participants and the study was considered of minimal risk to patients, the requirement of a full IRB process was largely waived according to the Ethical Principles and Guidelines for the Protection of Human Subjects of Research (the "Belmont Report") and the requirements of the Health Insurance Portability and Accountability Act (HIPAA) of 1996.

\section{Data collection details}

The cohorts for this study consisted of patients who presented to the Emergency Department with symptoms suspicious for COVID at the participating institutions:

Mass Gen Brigham affiliated hospitals (Mass General Hospital, Brigham and Women's Hospital, NewtonWellesley Hospital, North Shore Medical Center, Faulkner Hospital); Children's National Hospital in Washington, D.C.; NIHR Cambridge Biomedical Research Centre; The Self-Defense Forces Central Hospital in Tokyo; National Taiwan University MeDA Lab and MAHC and Taiwan National Health Insurance Administration; Tri-Service General Hospital in Taiwan; Kyungpook National University Hospital in South Korea; Faculty of Medicine, Chulalongkorn University in Thailand; Diagnosticos da America SA in Brazil; University of California, San Francisco; VA San Diego; University of Toronto; National Institutes of Health in Bethesda, Maryland; University of Wisconsin-Madison School of Medicine and Public Health; Memorial Sloan Kettering Cancer Center in New York; and Mount Sinai Health System in New York.

The inclusion criteria were: 1 . patient presented to the hospital's Emergency Department (ED) or equivalent, 2. patient had a PCR test done during the current hospitalization or had a COVID PCR test with a positive result prior to hospitalization, 3. patient had a CXR in the ED or during the hospital stay, 4. Patient's record had at least 5 of the EMR values (vitals, lab results and outcomes) detailed in Extended Data Table 1 obtained in the ED or during hospitalization.

The CXR and the EMR features used were the first available CXR and EMR values available for each patient obtained during this hospital stay. The datasets included COVID positive and COVID negative patients, determined by the PCR test. Client sites included all of their patients with a PCR positive test. Since most had more COVID negative than positive patients, we limited the number of negative patients included to at most $95 \%$ of the total cases at each client-site. In total, 21 EMR features were used as input to the model. The outcome (i.e., "ground truth") labels were assigned based on patient requirements after 24- and 72-hour periods from initial admission to the ED. A detailed list of the requested EMR features and outcomes can be seen in Extended Data Table 1. 
The variation of these features across different client-sites can be appreciated in Extended Data Fig.1. Data harmonization was not performed between different client-sites in order to train a robust model that could generalise well to unseen patient populations.

The distribution of oxygen treatment using different devices at different client-sites is shown in Extended Data Fig.2, which details the device usage at admission to the Emergency Department (ED), and after 24hour and 72-hour periods.

The number of positive COVID-19 cases, confirmed by a single PCR test, are listed in Extended Data Table 2. Each client-site was asked to randomly split its dataset into 3 parts, $70 \%$ for training, $10 \%$ for validation, and $20 \%$ for testing. The random splits were generated independently for each of the repeated three local and FL training and evaluation experiments for both $24 \mathrm{~h}$ and $72 \mathrm{~h}$ outcome prediction models.

\section{Feature imputation \& normalization}

A MissForest algorithm ${ }^{1}$ was used to impute EMR features, based on the local training dataset. If an EMR feature was completely missing from a client-site dataset, the mean value of that feature, calculated exclusively on data from MGB client-sites, was used. Then, EMR features were rescaled to zero-mean and unit-variance based on statistics calculated on data from the MGB client-sites.

\section{Details of the EMR-CXR data fusion}

To model the interactions of features from EMR and CXR data on a case-level, a deep feature scheme was used, based on Deep \& Cross network architecture ${ }^{2}$. Binary/categorical features for the EMR inputs, as well as 512-dimensional image features in the CXR, were transformed into fused dense vectors of real values by embedding and stacking layers. The transformed dense vectors served as input to the fusion framework, which specifically employed a crossing network to enforce fusion among input from different sources. The crossing network performed explicit feature crossing within its layers, by conducting inner products between the original input feature and output from the previous layer, thus increasing the degree of interaction across features. At the same time, two individual classic deep neural networks with several stacked fully-connected feed-forward layers were trained. The final output of our framework was then derived from the concatenation of both classic and crossing networks.

\section{CORISK model and derivation of clinical score}

Our preliminary, single-site patient outcome prediction model (calculating a risk score termed as "CORISK") was trained using the MGB COVID cohort consisting of over 7,000 patients with a positive or undetermined COVID status (at time of data collection). EMR data and CXR images of these patients were extracted from the Enterprise Data Warehouse (EDW) and clinical Picture Archiving and Communication System (PACS) systems during the period extending from March to May 2020. The CORISK model was validated using data from five hospitals within the MGB system, and cross-validated using different time periods during the study period. It achieved an average prediction accuracy of over 
$85 \%$. We further derived the clinical scores and the corresponding diagnostic criteria ("CORISK24" and "CORISK72", for 24- and 72-hours patient outcome assessment), similar to CORISK model's predictions. The clinical scores could be used by clinicians to triage patients into appropriate care settings.

The evaluation of the model is based on the average AUC of three prediction tasks derived from the CORISK score (LFO, HFO/NIV or MV). To compute it, we generate three sets of labels and predictions $L 1=$ $\left\{P_{\text {pred }} P_{g t}\right\}^{3} 0.25, L 2=\left\{P_{\text {pred }} P_{g t}\right\}^{3} 0.5$, and $L 3=\left\{P_{\text {pred }} P_{\text {gt }}\right\}^{3} 0.5$, where $P_{\text {pred }}$ is the models CORISK predictions and $P_{g t}$ is the ground truth CORISK scores representing a specific oxygen treatment as described above for a client-site's test set. The average AUC was then computed as AUC $=1 / 3$ * (auc $(L 1)+$ $\operatorname{auc}(L 2)+\operatorname{auc}(L 3))$.

\section{Federated learning details}

A pseudo-algorithm of FL is shown in Extended Data Algorithm 1. In our experiments, we set the number of federated rounds to be $T=200$, with one local training epoch per round $t$ at each client. The number of clients $K$ was up to 20 , depending on the network connectivity of clients or available data for a specific targeted outcome period ( $24 \mathrm{~h}$ or $72 \mathrm{~h}$ ). The number of local training iterations $\mathrm{nk}$ depends on the dataset size at each client $k$ and is used to weigh each client's contributions when aggregating the model weights in FederatedAveraging. During FL, each client-site selects its best local model by tracking the model's performance on its local validation set. At the same time, the server determines the best global model based on the average validation scores sent from each client-site to the server after each FL round. After the FL training finishes, the best local models and best global model are automatically shared with all client-sites and evaluated on their local test data.

When training on local data only (the baseline), we set the epoch number to 200. The Adam optimizer was used for both local training and FL with an initial learning rate of 5e-5 and a stepwise learning rate decay with a factor 0.5 after every 40 epochs, which is important for the convergence of FederatedAveraging ${ }^{3}$. Random affine transformations, including rotation, translations, shear, scaling, and random intensity noise and shifts were applied to the images for data augmentation during training.

Due to the sensitivity of batch normalization (BN) layers ${ }^{4}$ when dealing with different clients in a nonindependent and identically distributed (non-IID) setting ${ }^{5}$, we found the best model performance to occur when keeping the pre-trained ResNet34 with spatial attention ${ }^{6}$ parameters fixed during FL (i.e. using a learning rate of zero for those layers). The Deep \& Cross network that combines image features with the EMR features does not contain BN layers and hence was not affected by BN's instability issues.

In this study, we investigated a privacy-preserving scheme that shares only partial model updates between server and client-sites. To be exact, the weight updates (aka. gradients) were shared only if their absolute value was above a certain percentile threshold $t_{k}{ }^{(t)}$ (Fig. 4), which was computed from all nonzero gradients DWk(t) and could be different for each client $k$ in each $\mathrm{FL}$ round $t$. Variations of this 
scheme could include additional clipping of large gradients or differential privacy schemes ${ }^{7}$ that add random noise to the gradients or even to the raw data before feeding it to the network ${ }^{7,8}$.

\section{Statistical analysis}

We conducted a Wilcoxon signed-rank test to confirm the significance of the observed improvement in performance between the locally trained model and the FL model for the 24 and $72 \mathrm{hr}$ time point (see Fig. 3 and Extended Data Fig. 6). The null hypothesis was rejected with a one-sided p-value $<<1 \mathrm{e}-3$ in both cases.

A Pearson's correlation was used to assess the generalisability (robustness to other client-sites' test data) of locally trained models in relation to respective local dataset size. Only a moderate correlation was observed $(r=0.43, p=0.035, d f=17$ for the $24 \mathrm{~h}$ model and $r=0.62, p=0.003, d f=16$ for the $72 \mathrm{~h}$ model). This indicates that dataset size alone is not the only factor in determining a model's robustness to unseen data.

To compare the ROC curves from different sites and FL global one (shown in Fig. 5), we bootstrapped 1000 replicates from the data and computed their AUCs. We standardized the difference $D=(A U C 1-$ AUC2)/s, where $s$ is the standard deviation of the bootstrap differences and AUC1 and AUC2 the AUC of the two (original) ROC curves. By comparing D with normal distribution, we obtained the significance $p$ values illustrated in Table 4. With alternative hypotheses to be FL greater than the compared one, most of the $p$-values give very small values, indicating the statistical significance of FL outcomes. Computation of $p$-values was conducted in R with the pROC library ${ }^{9}$.

\section{Benefits to client-sites with small datasets}

We compared locally trained models with the global FL model on each client's test data. For a client-site with a relatively small dataset, there are two typical ways to get a model: one is to train locally with its own data, the other is to apply a model trained on a larger dataset. It is shown in Extended Data Fig. 5 that these two ways are outperformed on all three tasks by the FL model significantly, indicating that the benefit for client-sites with small datasets is huge.

Another particular site (client-site 16) had an unbalanced dataset, with most subjects being of mild disease severity and with only a few severe cases. Thus, the improvement in prediction accuracy for the category with few cases was substantial; see Extended Data Fig. 3, $t>=0.5$ (categories $>=$ high-flow oxygen device). The FL model achieved a higher true positive rate for the two positive (severe) cases at a markedly lower false positive rate compared to the local model, both shown in the receiver operating characteristic (ROC) plots and confusion matrices. The difference in dataset distribution for the two compared client-sites can be seen in Extended Data Fig. 4.

\section{Effect of different demographics}


To investigate the effectiveness of our model on patients with different demographics, especially with different races, we test our model on the test set of 5 client-sites in the Boston area and show results for different race populations accordingly. The results of Black or African American and White or Caucasian population (We don't show results for other races here due to the limited sample sizes) is shown in the Extended Data Table 3. We show the mean and the standard deviation of AUCs of the 5 local models and the AUC for the federated trained model on 3 tasks for both 24- and 72-h prediction. We can see that the improvement brought by federated training is consistent across different races.

\section{Effect of different COVID-19 status}

Extended Data Fig. 6 shows the performance of our model in predicting oxygen treatment in 24/72h for COVID positive/negative patients respectively. The COVID status is determined by the PCR tests performed at the visit of ED. It can be shown that our model is robust to both COVID positive and negative patients. This is crucial for our model to be applied on all the patients to support their triage since the PCR test results are usually not available at the time of ED disposition.

\section{Limitations and areas for future research}

The study found the global models (see under 'Federated Learning Details') to be more robust compared to locally trained models when assessed across all client-sites' test data. Locally optimized models might provide improved performance on a client-site's own test data, but usually resulted in a loss of generalisability. Local model selection always depends on the local validation set's quality and how well it represents the real test data's characteristics. In contrast, the global model selected based on the averaged validation scores from each client-site turns out to have better generalisability.

It is possible to achieve higher-performing models on a local dataset when tuning the training strategies more exhaustively ${ }^{10}$, such as varying data augmentation, learning rate schedule, and data sampling methods. However, generalisability to other sites' data is still expected to be limited due to the lack of representative training data. Future approaches may incorporate automated hyperparameter searching ${ }^{10}$, neural architecture search ${ }^{11}$, and other automated machine learning (AutoML) ${ }^{12}$ approaches to find the optimal training parameters for each client-site more efficiently.

Slow or interrupted internet connectivity sometimes caused some clients' model updates to be not included in each round of FL training. Such clients are commonly known as "stragglers" ${ }^{13}$. Future implementations of FL might specifically address this issue by allowing asynchronous updates ${ }^{14}$.

Known issues of $\mathrm{BN}$ in $\mathrm{FL}^{4}$ motivated us to fix our base model for image feature extraction ${ }^{6}$ in order to reduce the divergence between unbalanced client-sites. Future work might explore different types of normalization techniques in order to allow the training of Al models in FL more effectively when the clients' data is non-IID. 
Although privacy is a key concern for participants of $\mathrm{FL}$, the actual quantification of data leakage during model training is still rather unexplored as most efforts revolve around IT security for the communication between participants and server. Future work could aim to quantify the amount of data leakage that is still recoverable by model inversion methods or attacks on the gradients. A quantifiable way to measure privacy would allow better choices for deciding the minimal privacy parameters necessary while maintaining clinically acceptable performance $7,8,15$.

A final, but important limitation to all machine learning models is that they are limited by the quality of the training data. Institutions interested in deploying these algorithms for clinical care need to understand the inherent biases in the training. For example, the ground truth data used in the training of the EXAM model was 24- and 72-hour oxygen consumption in the patient. It is assumed that the oxygen consumption is the oxygen need. However, in the early period of the COVID-19 pandemic, many patients were provided high flow oxygen prophylactically, regardless of their oxygen need. Such clinical practice could skew the oxygen need predictions made by this model.

\section{Methods References}

1. Stekhoven, D. J. \& Bühlmann, P. MissForest-non-parametric missing value imputation for mixed-type data. Bioinformatics 28, 112-118 (2012).

2. Wang, R., Fu, B., Fu, G. \& Wang, M. Deep \& Cross Network for Ad Click Predictions. Proceedings of the $A D K D D^{\prime} 17$ on $Z Z Z$ - $A D K D D^{\prime} 17$ (2017) doi:10.1145/3124749.3124754.

3. Li, X., Huang, K., Yang, W., Wang, S. \& Zhang, Z. On the Convergence of FedAvg on Non-IID Data. arXiv [stat.ML] (2019).

4. loffe, S. \& Szegedy, C. Batch Normalization: Accelerating Deep Network Training by Reducing Internal Covariate Shift. arXiv [cs.LG](2015).

5. Hsieh, K., Phanishayee, A., Mutlu, O. \& Gibbons, P. B. The Non-IID Data Quagmire of Decentralized Machine Learning. arXiv [cs.LG](2019).

6. Zhong, A. et al. Deep Metric Learning-based Image Retrieval System for Chest Radiograph and its Clinical Applications in COVID-19. arXiv [eess.IV] (2020).

7. Li, W. et al. Privacy-Preserving Federated Brain Tumour Segmentation. Machine Learning in Medical Imaging 133-141 (2019) doi:10.1007/978-3-030-32692-0_16.

8. Kaissis, G. A., Makowski, M. R., Rückert, D. \& Braren, R. F. Secure, privacy-preserving and federated machine learning in medical imaging. Nature Machine Intelligence vol. 2 305-311 (2020).

9. Robin, X. et al. pROC: an open-source package for R and S+ to analyze and compare ROC curves. BMC Bioinformatics 12, 77 (2011).

10. Yang, D. et al. Searching Learning Strategy with Reinforcement Learning for 3D Medical Image Segmentation. in Medical Image Computing and Computer Assisted Intervention - MICCAI 20193 11 (Springer International Publishing, 2019).

11. Elsken, T., Metzen, J. H. \& Hutter, F. Neural Architecture Search: A Survey. arXiv [stat.ML] (2018). 
12. Yao, Q. et al. Taking Human out of Learning Applications: A Survey on Automated Machine Learning. arXiv [cs.Al] (2018).

13. Yang, Q., Liu, Y., Chen, T. \& Tong, Y. Federated Machine Learning: Concept and Applications. (2019).

14. Xie, C., Koyejo, S. \& Gupta, I. Asynchronous Federated Optimization. arXiv [cs.DC] (2019).

15. Rieke, N. et al. The future of digital health with federated learning. NPJ Digit Med 3, 119 (2020).

16. McMahan, B., Moore, E., Ramage, D., Hampson, S. \& y Arcas, B. A. Communication-Efficient Learning of Deep Networks from Decentralized Data. in (eds. Singh, A. \& Zhu, J.) vol. 54 1273-1282 (PMLR, 2017).

17. NVIDIA Clara Imaging. https://developer.nvidia.com/clara-medical-imaging.

\section{End Notes}

\section{Acknowledgements}

The views expressed in this study are those of the authors and not necessarily those of the NHS, the NIHR, the Department of Health and Social Care or any of the organizations associated with the authors.

MGB would like to acknowledge the following individuals for their support: James Brink MD, Department of Radiology, Massachusetts General Hospital, Harvard Medical School, Boston, MA;

Mannudeep Kalra MD, Department of Radiology, Massachusetts General Hospital, Harvard Medical School, Boston, MA; Nir Neumark MD, MSc., Center for Clinical Data Science, Massachusetts General Brigham, Boston, MA; Thomas Schultz, Department of Radiology, Massachusetts General Hospital, Boston, MA; Ning Guo, Center for Advanced Medical Computing and Analysis, Department of Radiology, Massachusetts General Hospital, Harvard Medical School, Boston, MA; Jayashree Kalpathy Cramer PhD, Director, QTIM lab at the Athinoula A. Martinos Center for Biomedical Imaging at MGH; Stuart Pomerant, Department of Radiology, Massachusetts General Hospital, Harvard Medical School, Boston, MA; Giles Boland MD, Department of Radiology, Brigham and Women's Hospital, Harvard Medical School, Boston, MA; William Mayo-Smith MD, Department of Radiology, Brigham and Women's Hospital, Harvard Medical School, Boston, MA

UCSF would like to acknowledge Peter B. Storey, Jed Chan and Jeff Block for implementing the UCSF FL client infrastructure and Wyatt Tellis, $\mathrm{PhD}$ for providing the source imaging repository for this work. The UCSF EMR and clinical notes for this study were accessed via the COVID-19 Research Data Mart https://data.ucsf.edu/covid19.

Faculty of Medicine, Chulalongkorn University_would like to acknowledge the Ratchadapisek Sompoch Endowment Fund RA (PO) 001/63 for the Collection and Management of COVID-19 Related Clinical Data and Biological Specimens for Research Task Force, Faculty of Medicine, Chulalongkorn University. 
NIHR Cambridge Biomedical Research Centre would like to acknowledge that Andrew Priest is supported by the National Institute for Health Research (Cambridge Biomedical Research Centre at the Cambridge University Hospitals NHS Foundation Trust).

National Taiwan University MeDA Lab and MAHC and Taiwan National Health Insurance Administration would like to acknowledge MOST Joint Research Center for AI Technology and All Vista Healthcare (AINTU)

National Health Insurance Administration, Taiwan and Ministry of Science and Technology, Taiwan National Center for Theoretical Sciences Mathematics Division.

National Institutes of Health $(\mathrm{NIH})$. would like to acknowledge that The National Institutes of Health $(\mathrm{NIH})$ Medical Research Scholars Program is a public-private partnership supported jointly by the $\mathrm{NIH}$ and generous contributions to the Foundation for the NIH from the Doris Duke Charitable Foundation, the American Association for Dental Research, the Colgate-Palmolive Company,Genentech, alumni of student research programs, and other individual supporters via contributions to the Foundation for the National Institutes of Health.

\section{Author information}

These authors contributed equally: Ittai Dayan, Holger Roth, Aoxiao Zhong, Fiona J Gilbert, Quanzheng Li, Mona G. Flores

Affiliations

\section{MGH Radiology and Harvard Medical School, Boston, MA, USA}

Ittai Dayan

\section{NVIDIA, Santa Clara, CA, USA}

Holger Roth, Ahmed Harouni, Anas Abidin, Andrew Liu, CK Lee, Colleen Ruan, Daguang Xu, Eddie Huang, Griffin Lacey, Jesse Tetreault, iahui Guan, Kristopher Kersten, Nicola Rieke, Pedro Mario Cruz e Silva, Mona G. Flores, Abood Quraini, Andrew Feng, Colin Compas, Deepeksha Bhatia, Isaac Yang, Mohammad Adil \& Yuhong Wen

\section{Center for Advanced Medical Computing and Analysis, Department of Radiology, Massachusetts General Hospital, Harvard Medical School, Boston, MA, USA}

Aoxiao Zhong, Dufan Wu, Hui Ren,Xiang Li \& Quanzheng Li

\section{San Diego VA Health Care System, San Diego, CA, USA}

Amilcare Gentili 
5. Department of Neurosurgery, Icahn School of Medicine at Mount Sinai, New York, NY, USA

Anthony Beardsworth Costa \& Young Joon Kwon

6. Radiology \& Imaging Sciences / Clinical Center, National Institutes of Health, Bethesda, MD, USA

Bradford J. Wood

7. Division of Cardiovascular Surgery, Department of Surgery, Tri-Service General Hospital, National Defense Medical Center, Taipei, Taiwan, R.O.C.

Chien-Sung Tsai

8. Department of Otolaryngology-Head and Neck Surgery, Tri-Service General Hospital, National Defense Medical Center, Taipei, Taiwan, R.O.C. and Graduate Institute of Medical Sciences, National Defense Medical Center, Taipei, Taiwan, R.O.C.

Chih-Hung Wang

9. Center for Research in Biological Systems, University of California, San Diego, CA, USA

Chun-Nan Hsu

10. Diagnósticos da América SA (DASA), Brazil

Felipe Campos Kitamura, Gustavo César de Antônio Corradi, Matheus Ribeiro Furtado de Mendonça \& Vitor de Lima Lavor

11. Division of Pediatric Pulmonary and Sleep Medicine, Children's National Hospital, Washington, DC, USA

Gustavo Nino

12. Memorial Sloan Kettering Cancer Center, New York, NY, USA

Hao-Hsin Shin, Krishna Juluru, Krishna Nand Keshava Murthy, Natalie Gangai \& Pierre Elnajjar

13. Self-Defense Forces Central Hospital, Tokyo, Japan

Hirofumi Obinata, Shuichi Kawano, Hisashi Sasaki, Hitoshi Mori \& Tatsuya Kodama

14. Center for Intelligent Imaging, 2Department of Radiology and Biomedical Imaging, University of California, San Francisco, California, USA

Jason C. Crane, Pablo F. Damasceno, Christopher P. Hess, Jae Ho Sohn \& Sharmila Majumdar 
15. Departments of Radiology and Medical Physics, The University of Wisconsin-Madison School of Medicine and Public Health, Madison, WI, USA

John W. Garrett

16. Department of Radiology, NIHR Cambridge Biomedical Resource Centre, University of Cambridge, Cambridge, UK

Josh D Kaggie, Fiona J Gilbert \& Sarah Hickman

17. Department of Internal Medicine, Yeungnam University College of Medicine, Daegu, South Korea Jung Gil Park \& Min Kyu Kang

18. Center for Clinical Data Science, Massachusetts General Brigham, Boston, MA, USA

Keith Dreyer, Marcio Rockenbach, Varun Buch \& Bernardo Bizzo, Evan Leibovitz

19. Sheikh Zayed Institute for Pediatric Surgical Innovation, Children's National Hospital, Washington, DC, USA

Carlos Tor Diez \& Marius George Linguraru

20. Joint Dept. of Medical Imaging, Sinai Health System, University of Toronto, Toronto, Canada and Lunenfeld-Tanenbaum Research Institute, Toronto, Canada

Masoom A. Haider

21. Lunenfeld-Tanenbaum Research Institute, Toronto, Canada

Meena AbdelMaseeh

22. MeDA Lab and Institute of Applied Mathematical Sciences, National Taiwan University, Taipei, Taiwan

Pochuan Wang \& Weichung Wang

23. Center for Interventional Oncology, National Institutes of Health, Bethesda, MD, USA

Sheng Xu \& Sheridan Reed

24. Research Affairs, Faculty of Medicine, Chulalongkorn University, Bangkok, Thailand, Center for Artificial Intelligence in Medicine, Faculty of Medicine, Chulalongkorn University, Bangkok, Thailand

Sira Sriswasdi 
25. Department of Internal Medicine, School of Medicine, Kyungpook National University, Daegu, South Korea

Soo Young Park, Won Young Tak \& Yu Rim Lee

26. Departments of Radiology, Medical Physics, and Biomedical Engineering, The University of Wisconsin-Madison School of Medicine and Public Health, Madison, WI, USA

Thomas M. Grist

27. Department of Pediatrics, Faculty of Medicine, Chulalongkorn University, Bangkok, Thailand and Thai Red Cross Emerging Infectious Diseases Clinical Center, King Chulalongkorn Memorial Hospital, Bangkok, Thailand

Watsamon Jantarabenjakul \& Thanyawee Puthanakit

28. Medical Review and Pharmaceutical Benefits Division, National Health Insurance Administration, Taipei. Taiwan

Weichung Wang \& Chiu-Ling Lai

29. Harvard T.H. Chan School of Public Health, Harvard University, Boston, MA, USA

Xihong Lin

30. Department of Radiology, NIHR Cambridge Biomedical Resource Centre, Cambridge University Hospital. Cambridge, UK

Andrew N Priest

31. Department of Radiology and Imaging Sciences, National Institutes of Health, Bethesda, MD, USA and National Cancer Institute, National Institutes of Health, Bethesda, MD, USA

\section{Baris Turkbey}

32. Hasso Plattner Institute for Digital Health at Mount Sinai, Icahn School of Medicine at Mount Sinai and Department of Genetics and Genomic Sciences, Icahn School of Medicine at Mount Sinai, New York, NY, USA

Benjamin Glicksberg

33. Department of Internal Medicine, Catholic University of Daegu School of Medicine, Daegu, South Korea

Byung Seok Kim 
34. Planning and Management Office, Tri-Service General Hospital, National Defense Medical Center, Taipei, Taiwan, R.O.C.

\section{Chia-Jung Hsu \& Chia-Cheng Lee}

35. School of Medicine, National Defense Medical Center, Taipei, Taiwan, R.O.C. and School of Public Health, National Defense Medical Center, Taipei, Taiwan, R.O.C. and Graduate Institute of Life Sciences, National Defense Medical Center, Taipei, Taiwan, R.O.C.

\section{Chin Lin}

36. Department of Neurosurgery, NYU Grossman School of Medicine, New York, NY, USA

\section{Eric K Oermann}

37. MOST/NTU All Vista Healthcare Center, Center for Artificial Intelligence and Advanced Robotics, National Taiwan University, Taipei, Taiwan

\section{Li-Chen Fu}

38. Division of General Internal Medicine and Geriatrics (Fralick), Sinai Health System, Toronto, Canada Mike Fralick

39. Department of Computer Engineering, Faculty of Engineering, Chulalongkorn University, Bangkok, Thailand

\section{Peerapon Vateekul}

40. Schwartz/Reisman Emergency Medicine Institute, Sinai Health, Toronto, ON, Canada and Department of Family and Community Medicine, University of Toronto, Toronto, ON, Canada

Shelley L. McLeod

41. Department of Medicine, NIHR Cambridge Biomedical Resource Centre, University of Cambridge, Cambridge, UK

\section{Stefan Graf}

42. National Cancer Institute, National Institutes of Health, Bethesda, MD, USA and Clinical Research Directorate, Frederick National Laboratory for Cancer, National Cancer Institute. Frederick, MD, USA

43. Department of Microbiology, Sinai Health/University Health Network, Toronto, Canada and Department of Laboratory Medicine and Pathobiology, University of Toronto, Toronto. Canada Public 


\section{Health Ontario Laboratories, Toronto, Canada}

Tony Mazzulli

\section{Chulalongkorn University Biomedical Imaging Group and Division of Nuclear Medicine, Department of Radiology, Faculty of Medicine, Chulalongkorn University, Bangkok, Thailand}

Yothin Rakvongthai

\section{Contributions}

Ittai Dayan and Mona G. Flores contributed to the acquisition of the data, study support, drafting and revising the manuscript, study design, study conception, and analysis and interpretation of the data; Holger Roth, Aoxiao Zhong and Quanzheng Li, contributed to the acquisition of the data, study support, drafting and revising the manuscript, study design, and analysis and interpretation of the data; Fiona J Gilbert contributed to the acquisition of the data, study support, drafting and revising the manuscript; Jiahui Guan contributed to the support of the study, drafting and revising the manuscript, and analysis and interpretation of the data; Varun Buch contributed to the acquisition of the data, study support and study design; Daguang Xu contributed to the acquisition of the data, study support, drafting and revising the manuscript, and analysis and interpretation of the data; Anthony Beardsworth Costa, Bradford J. Wood, John W. Garrett and Krishna Juluru contributed to the acquisition of the data and drafting, and revising the manuscript; Nicola Rieke, contributed to the support of the study, and drafting and revising the manuscript; Ahmed Harouni, Anas Abidin, Andrew Liu, CK Lee, Colleen Ruan, Eddie Huang, Griffin Lacey, Jesse Tetreault, Kristopher Kersten, Pedro Mario Cruz e Silva, Abood Quraini, Andrew Feng, Colin Compas, Deepeksha Bhatia, Isaac Yang, Mohammad Adil and Yuhong Wen contributed to the support of the study; Amilcare Gentili, Chien-Sung Tsai, Chih-Hung Wang, Chun-Nan Hsu, Dufan Wu, Felipe Campos Kitamura, Gustavo César de Antônio Corradi, Gustavo Nino, Hao-Hsin Shin, Hirofumi Obinata, Hui Ren, Jason C. Crane, Josh D Kaggie, Jung Gil Park, Keith Dreyer, Marcio Rockenbach, Marius George Linguraru, Masoom A. Haider, Meena AbdelMaseeh, Pablo F. Damasceno, Pochuan Wang, Sheng Xu, Shuichi Kawano, Sira Sriswasdi, Soo Young Park, Thomas M. Grist, Watsamon Jantarabenjakul, Weichung Wang, Won Young Tak, Xiang Li, Xihong Lin, Young Joon Kwon, Andrew N Priest, Baris Turkbey, Benjamin Glicksberg, Bernardo Bizzo, Byung Seok Kim, Carlos Tor Diez, ChiaCheng Lee, Chia-Jung Hsu, Chin Lin, Chiu-Ling Lai, Christopher P. Hess, Eric K Oermann, Evan Leibovitz, Hisashi Sasaki, Hitoshi Mori, Jae Ho Sohn, Krishna Nand Keshava Murthy, Li-Chen Fu, Matheus Ribeiro Furtado de Mendonça, Mike Fralick, Min Kyu Kang, Natalie Gangai, Peerapon Vateekul, Pierre Elnajjar, Sarah Hickman, Sharmila Majumdar, Shelley L. McLeod, Sheridan Reed, Stefan Graf, Stephanie Harmon, Tatsuya Kodama, Thanyawee Puthanakit, Tony Mazzulli, Vitor de Lima Lavor, Yothin Rakvongthai and Yu Rim Lee contributed to the acquisition of the data. 


\section{Ethics declarations}

\section{Competing interests}

This study was organized and coordinated by NVIDIA. Y.W., M.A., I.Y., A.Q., C.C., D.B., A.F., H.R., J.G., D.X., N.R., A.H., K.K., C.R., A.A., C.K.L, E.H., A.L., G.L., P.M.C.S, J.T., and M.G.F. are employees of NVIDIA and own stock as part of the standard compensation package.

J.G. declared ownership of NVIDIA Stock.

C.H. declared Research travel, Siemens Healthineers AG; Conference Travel, EUROKONGRESS; GmBH; and Personal fees (Consultant, GE Healthcare LLC; DSMB Member, Focused Ultrasound Foundation).

F.J.G declared research collaborations with Merantix, Screen-Point, Lunit and Volpara, GE Healthcare and undertakes paid consultancy for Kheiron and Alphabet.

M.L. declared that he is the co-founder of PediaMetrix Inc. and is on the Board of the SIPAIM Foundation

S.E.H declared research collaborations with Merantix, Screen-Point, Lunit and Volpara.

B.J.W and S.X. declared that NIH and NVIDIA have a Cooperative Research and Development Agreement. This work was supported (+/- in part) by the NIH Center for Interventional Oncology and the Intramural Research Program of the National Institutes of Health, via intramural NIH Grants Z1A CL040015, 1ZIDBC011242. Work supported by the NIH Intramural Targeted Anti-COVID-19 (ITAC) Program, funded by the National Institute of Allergy and Infectious Diseases. NIH may have intellectual property in the field.

\section{Additional information}

Correspondence and requests for materials should be addressed to Mona G. Flores, MD.

Reprints and permissions information is available at www.nature.com/reprints.

Data Availability: The authors declare that all of the analysis data supporting the findings of this study is available within the paper. The patient data used for local and federated training is not publicly available as it governed by regulatory restrictions such as HIPAA. The patient data was only visible locally to the participating sites and was not visible to any of the other participants.

\section{Figures}



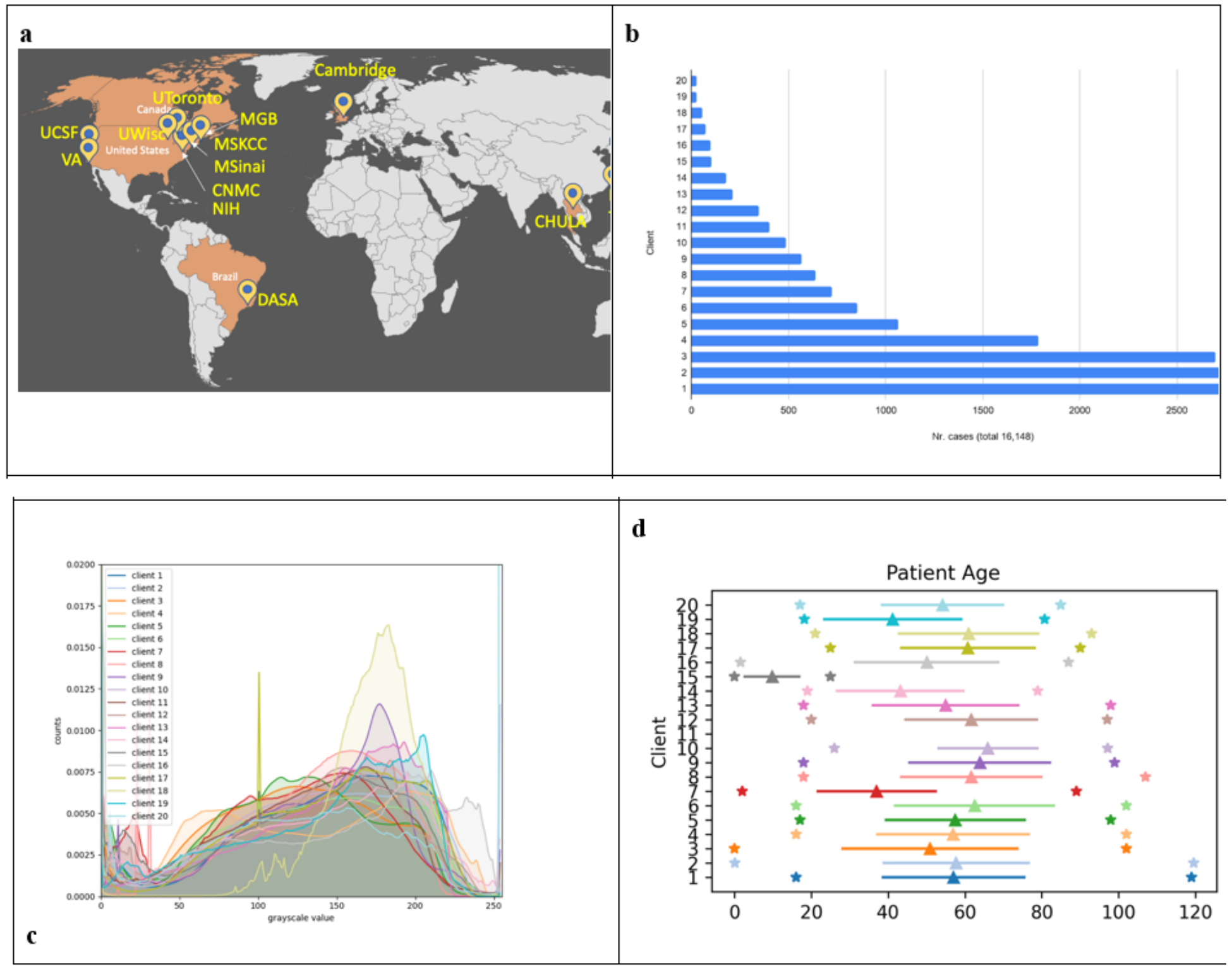

\section{Figure 1}

Data used in the EXAM Federated Learning study. a, EXAM included 20 different sites from around the globe. b, Number of cases that each institution or site contributed (client 1 being the largest site) $c$, CXR intensity distributions at each client site $d$, Age of patients included at each client-site showing the min. and max. ages (asterisks) and mean and standard deviation (length of bars). Note: The designations employed and the presentation of the material on this map do not imply the expression of any opinion whatsoever on the part of Research Square concerning the legal status of any country, territory, city or area or of its authorities, or concerning the delimitation of its frontiers or boundaries. This map has been provided by the authors. 


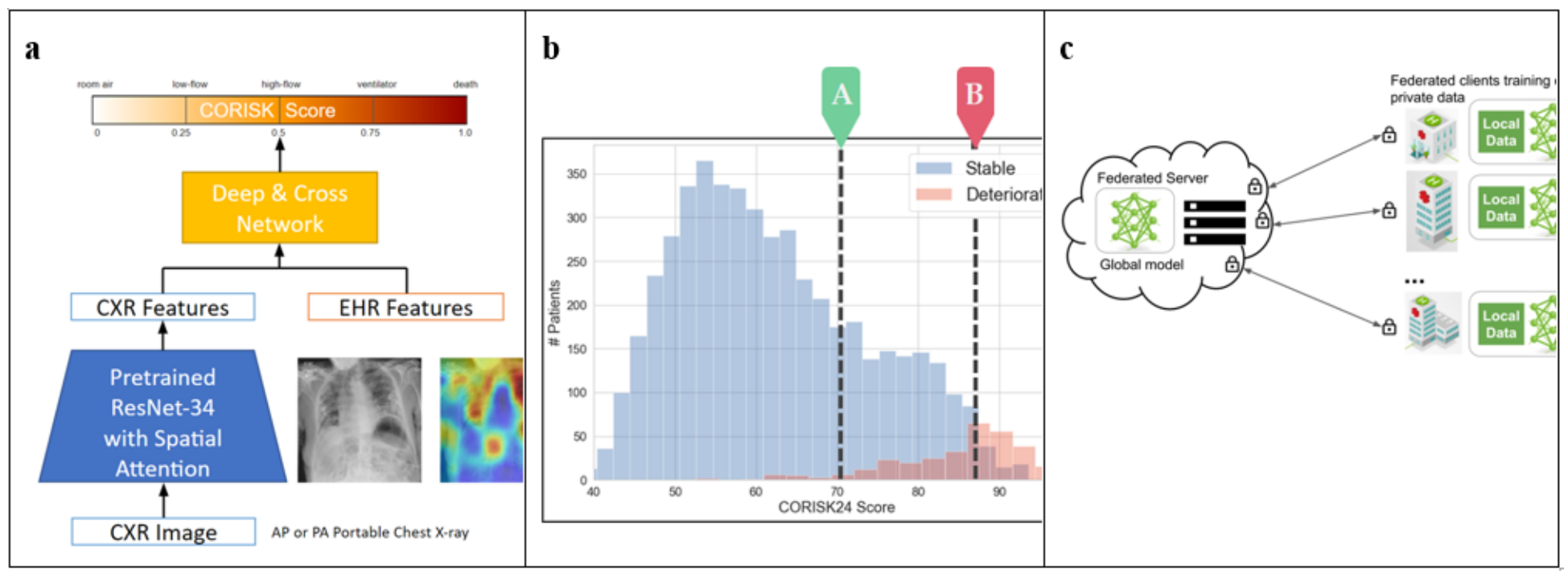

\section{Figure 2}

Description of the EXAM Federated Learning study. a, Proposed model to predict a COVID risk score. b, Histogram of CORISK results at MGB, with an illustration of how the score can be used for patient triage, in which ' $A$ ' is an example threshold for safe discharge that has $99.5 \%$ negative predictive value, and ' $B$ ' is an example threshold for Intensive Care Unit (ICU) admission that has $50.3 \%$ positive predictive value. C, Federated Learning using a client-server setup. 


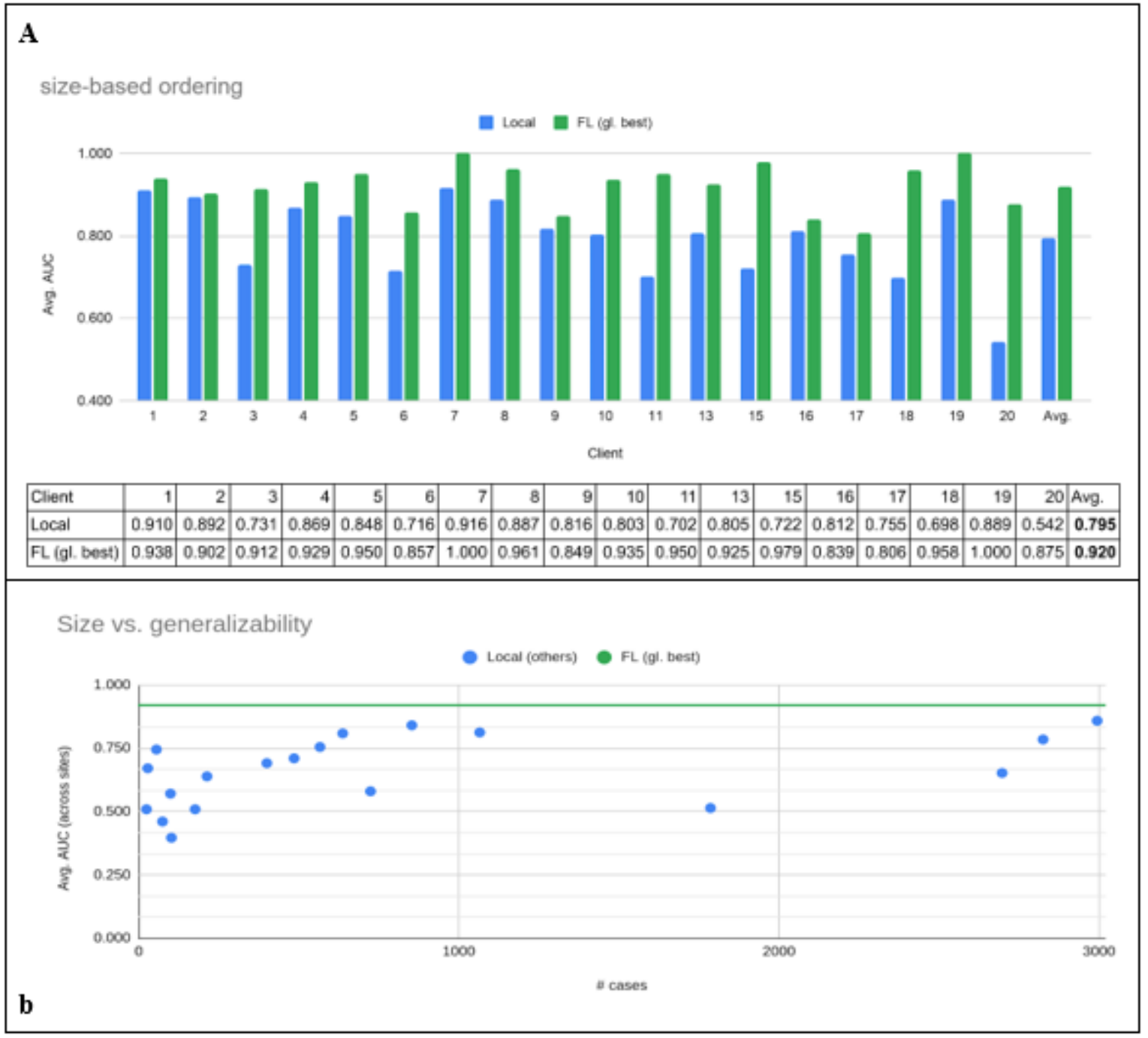

Figure 3

Federated Learning vs. local training performance. a, Test performance of models predicting $24 \mathrm{~h}$ oxygen treatment trained on local data only (Local) versus the performance of the best global model available on the server (FL (gl. best)). b, Generalisability (average performance on other sites' test data) as a function of a client's dataset size (\# cases). The average performance improved by $16 \%$ compared to locally trained models alone, while average generalisability of the global model improved by $38 \%$. Note, we show the performance for 18 of 20 clients here as client 12 had only outcomes for 72 hours (see Extended Data Fig. 7) and client 14 only cases with room air treatment, resulting in the evaluation metric (avg. AUC) being not applicable (see Methods). 


\section{Privacy-preserving FL}

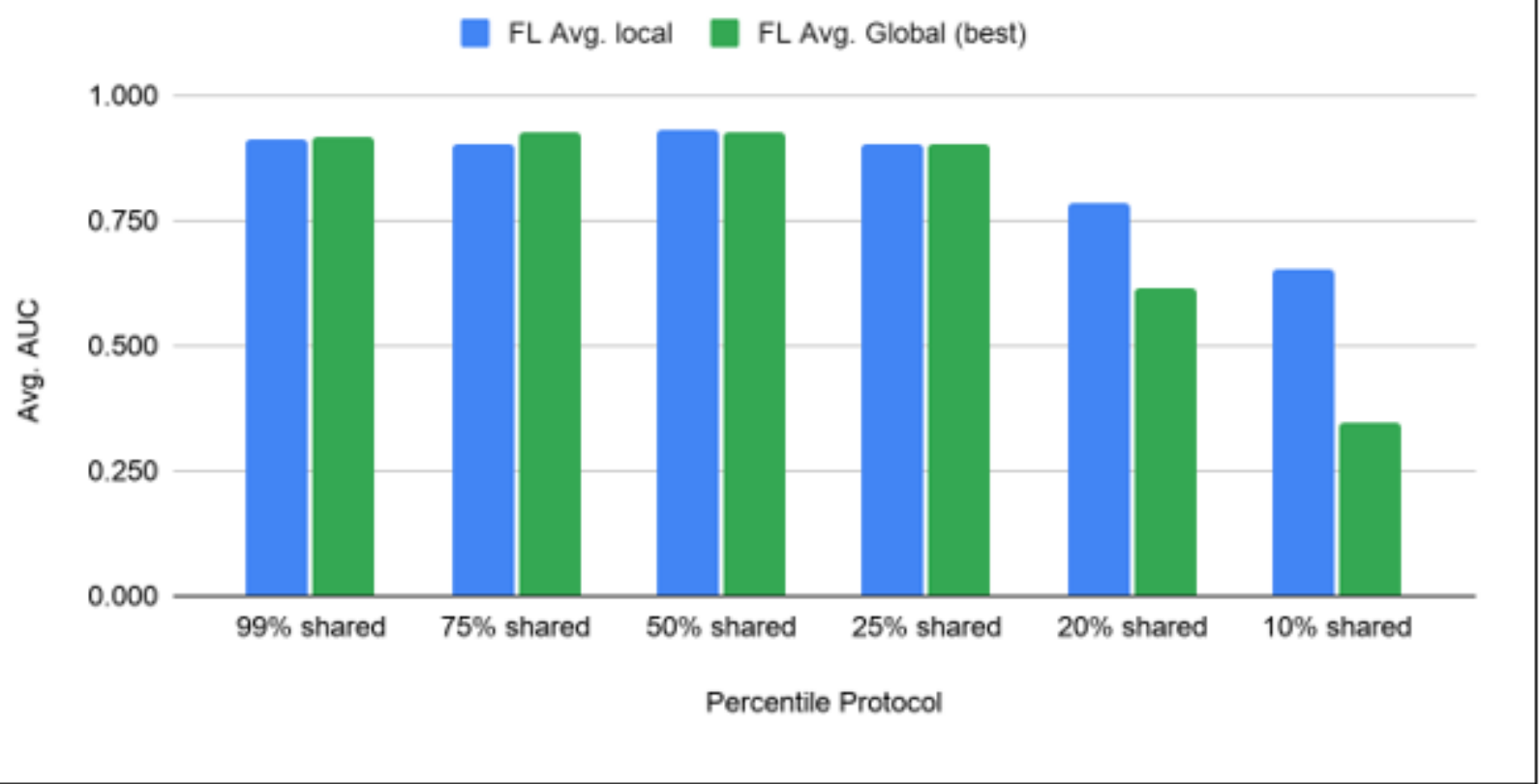

\section{Figure 4}

Safety enhancing features used in EXAM. Additional data-safety-enhancing features were assessed by only sharing a certain percentage of weight updates with the largest magnitudes before sending them to the server after each round of learning58. The figure shows that by using partial weight updates during $\mathrm{FL}$, models can be trained that reach a performance comparable to training while sharing the full information. This differential privacy technique decreases the risk for model inversion or reconstruction of the training image data through gradient interception.

\section{Supplementary Files}

This is a list of supplementary files associated with this preprint. Click to download.

- ExtendedFiguresandTables.docx 